\title{
Short-Packet Communications in Multi-Hop WPINs: Performance Analysis and Deep Learning Design
}

\author{
Toan-Van Nguyen*, Van-Dinh Nguyen ${ }^{\dagger}$, Daniel Benevides da Costa ${ }^{\ddagger \S}$ and Beongku An \\ * Dept. of Electrical and Computer Engineering, Utah State University, Logan, UT 84322-4120 USA \\ $\dagger$ SnT-Interdisciplinary Centre for Security, Reliability and Trust, University of Luxembourg, Luxembourg \\ $\ddagger$ Intelligent Wireless Communications (IWiCom) Research Group-Future Technology Research Center, \\ National Yunlin University of Science and Technology (YunTech), Douliu, Yunlin 64002, Taiwan, R.O.C. \\ $\S$ Dept. of Computer Engineering, Federal University of Ceará, Sobral 62010-560, CE, Brazil \\ I Dept. of Software and Communications Engineering, Hongik University, Republic of Korea \\ Emails: *vannguyentoan@gmail.com, †dinh.nguyen@uni.lu, ${ }^{\ddagger \S}$ danielbcosta@ ieee.org, ${ }^{\Uparrow}$ beongku@ hongik.ac.kr
}

\begin{abstract}
In this paper, we study short-packet communications (SPCs) in multi-hop wireless-powered Internet-of-Things networks (WPINs), where IoT devices transmit short packets to multiple destination nodes by harvesting energy from multiple power beacons. To improve system block error rate (BLER) and throughput, we propose a best relay-best user (bR-bU) selection scheme with an accumulated energy harvesting mechanism. Closed-form expressions for the BLER and throughput of the proposed scheme over Rayleigh fading channels are derived and the respective asymptotic analysis is also carried out. To support real-time settings, we design a deep neural network (DNN) framework to predict the system throughput under different channel settings. Numerical results demonstrate that the proposed bR-bU selection scheme outperforms several baseline ones in terms of the BLER and throughput, showing to be an efficient strategy for multi-hop SPCs. The resulting DNN can estimate accurately the throughput with low execution time. The effects of message size on reliability and latency are also evaluated and discussed.
\end{abstract}

Index Terms-Block error rate, deep neural network, energy harvesting, multi-hop IoT networks, short-packet communication, relay selection, ultra-reliable low-latency communications.

\section{INTRODUCTION}

Short-packet communication (SPC) plays an essential role in Internet-of-Things (IoT) and the fifth generation (5G) wireless networks that satisfies reliability and latency requirements [1]. SPCs were widely employed in high-speed trains, intelligent transportation, and factory automation, enabling ultrareliable low-latency communication (URLLC) services, for example, a reliability of $1-10^{-5}$ and latency of $1 \mathrm{~ms}$ for delivering a 32-byte packet [2]. To extend the lifetime of IoT devices, SPCs together with wireless energy transfer (WET) have been realized in IoT networks, thus introducing SPCbased green communications with stable energy supplies [3][5]. In [3], SPCs with WET were investigated for URLLC while the retransmission protocol was proposed for finitelength energy/information transfer systems in [4]. Resource allocation for IoT networks with WET was studied in [5] to improve the transmission reliability.

Recently, cooperative multi-hop relaying has received considerable attention with the aim of extending the radio coverage and improving the network performance [6], [7]. How- ever, in the context of SPC, the analysis has been restricted to dual-hop or single-hop transmissions, without any relay selection schemes [2], [3], [8]. Moreover, the impact of finite blocklength coding on multi-hop wireless-powered IoT networks, which brings several technical challenges related to the information-theoretic perspective, has not been well investigated in the literature.

In another front, deep neural network (DNN) has recently gained recognition as a viable solution to deal with various practical problems, such as queue management, resource allocation, and security problem in IoT systems and contemporary wireless networks [9]. Due to the accuracy in approximating high non-linear functions at considerably low-complexity, it has activated various interesting applications including prediction of secrecy outage probability, relay selection, and routing optimizations [9], [10]. DNN-based relay selection helps to expedite real-time settings in IoT networks since DNN models can precisely estimate desired performance metrics from high dimensional raw data even with dynamic environments and complex radio conditions. In this paper, we first study SPCs in multi-hop wireless-powered IoT networks (WPINs), where the best relay-best user (bR-bU) selection scheme is studied aiming to enhance the system performance, accompanied by a new deep learning evaluation approach. In a nutshell, we summarize the main contributions of this paper as follows:

- We propose the bR-bU selection scheme to improve the block error rate (BLER) and throughput in multi-hop WPINs, where the accumulate-then-transmit mechanism is employed for WET to enhance energy utilization.

- We derive new closed-form expressions for the BLER of bR-bU scheme, based on which the asymptotic BLER and throughput analysis are also carried out to further provide more insights into designs of the considered system setup.

- To support real-time settings, we design a DNN framework to predict the throughput of bR-bU scheme with high accuracy and low-latency inference process.

- Numerical results are provided to show that the bR-bU scheme outperforms several baseline ones in terms of BLER and throughput. It is also revealed a natural conflict 


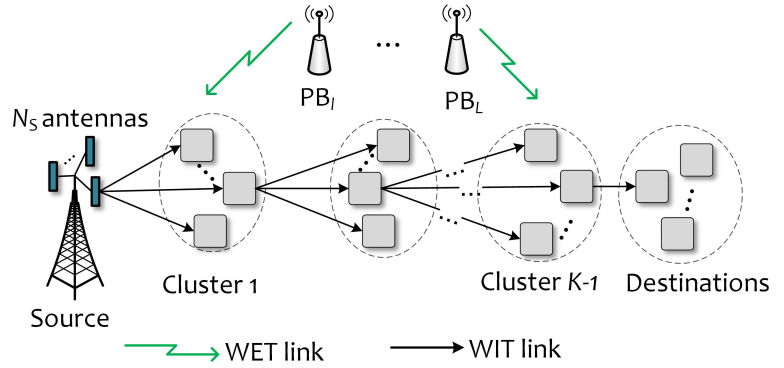

Fig. 1. The proposed multi-hop wireless-powered IoT network with SPC.

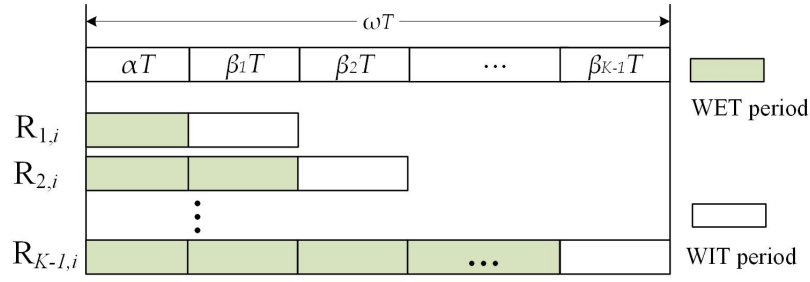

Fig. 2. Time block structure for accumulate-then-transmit WET and WIT.

between reliability and latency targets for fixed packet sizes.

Mathematical Notations and Functions: Boldface represents vector and $\|$.$\| symbolizes the Frobenius norm. \mathbb{E}\{\cdot\}$ and $(\cdot)^{H}$ are the expectation operator and transpose conjugate, respectively. $\Gamma($.$) and \gamma(.,$.$) represent the Gamma function$ [11, Eq. 8.310.1] and the lower incomplete Gamma function [11, Eq. (8.350.1)], respectively. $G_{a, b}^{c, d}[. \mid$.$] denotes the Meijers$ G-function [11, Eq. 9.301], and $K_{v}($.$) is the v$-order modified Bessel function of second kind [11, Eq. (8.432)].

\section{SYSTEM MODEL}

\section{A. System Description and Operation}

It is considered a multi-hop WPIN, where a source (S) with $N_{\mathrm{S}}$ antennas transmits data packets to multiple singleantenna destination (D) nodes via intermediate IoT devices located in $(K-1)$ clusters with $K \geq 2$, as shown in Fig. 1. It is assumed that each IoT device acts as a relay and uses decode-and-forward protocol to transmit short packets over multi-hop network. The relay, $\mathrm{R}_{k, n}$, is the $n$-th relay node, with $n=1, \ldots, N_{k}$, in the $k$-th cluster being a member of a set of relays $\mathcal{R}=\left\{\mathrm{R}_{k, n} \mid k=1, \ldots, K\right\}$, where $\mathrm{R}_{K, n} \equiv \mathrm{D}_{n}$. Since IoT devices have limited power supplies, they perform harvest energy harvesting from a set of power beacons $\mathcal{P}=$ $\left\{\mathrm{PB}_{l} \mid l=1, \ldots, L\right\}$. In this system, $\mathrm{S}$ can be considered as an access point and uses its own energy while single-antenna IoT devices are operated on half-duplex mode. We assume that all channels experience quasi-static independent identically distributed Rayleigh fading and perfect knowledge of channel state information (CSI) is available at each receiver terminal.

Consider a channel from $X$ to $\mathrm{Y}$, where $\mathrm{X} \in\left\{\mathrm{PB}_{l}, \mathrm{R}_{k, n}\right\}$ and $Y \in\left\{R_{k+1, j}, D_{n}\right\}$. Let $u_{X Y}$ be the channel coefficient of the $X \rightarrow Y$ link. The channel gain $\left|u_{X Y}\right|^{2}$ follows an exponential distribution, whose cumulative distribution function (CDF), $F_{\left|u_{X Y}\right|^{2}}(x)$, and probability density function (PDF), $f_{\left|u_{X Y}\right|^{2}}(x)$, are $F_{\left|u_{X Y}\right|^{2}}(x)=1-\exp \left(-\frac{x}{\lambda_{X Y}}\right)$ and $f_{\left|u_{X Y}\right|^{2}}(x)=\frac{1}{\lambda_{X Y}} \exp \left(-\frac{x}{\lambda_{X Y}}\right)$, respectively, where $\lambda_{\mathrm{XY}}$ is the mean of $\left|u_{X Y}\right|^{2}$. The large-scale pathloss can be modeled as $\lambda_{\mathrm{XY}}=\mathcal{L}\left(d_{\mathrm{XY}} / d_{0}\right)^{-\sigma_{\mathrm{PL}}}$ [7], where $d_{\mathrm{XY}}$ presents the distance from $\mathrm{X}$ to $\mathrm{Y}, \sigma_{\mathrm{PL}}$ is the pathloss exponent, $d_{0}$ is the reference distance, and $\mathcal{L}$ denotes the measured pathloss at $d_{0}$.

We consider short-packet transmission in multi-hop WPINs with two consecutive phases including WET and wireless information transfer (WIT), respectively [3], [4]. We employ $\omega$ channel uses for WET and WIT processes and each channel use occurs in period of $T$; hence, the total time for multihop transmission will be $\omega T$. Moreover, we denote by $\alpha$ the channel use for WET process such that WET consumes a period of $\alpha T$ and $(\omega-\alpha) T$ will be spent for WIT process, as sketched in Fig. 2. The time-division multiple access is adopted for WIT, where the period $(\omega-\alpha) T$ is equally divided into $K$ hops, with $\beta_{k}=(\omega-\alpha) / K, k=1, \ldots, K$, being the channel uses of each hop transmission. To enhance the WET capability at each relay, the accumulate-then-transmit WET protocol is employed, where the last relays have more chances to continuously harvest and accumulate energy before turning to data transmission. The last time period $\beta_{k} T$ at relay $\mathrm{R}_{k, i}$ is always reserved for its data transmission. Without loss of generality, the total time period of harvesting and accumulating energy at $\mathrm{R}_{k, i}$ can be expressed as $\alpha T+\sum_{m=1}^{k-1} \beta_{m} T$. Thus, the harvested energy at $\mathrm{R}_{k, i}$ from $L$ PBs can be expressed as

$$
E_{k, i}=\sum_{l=1}^{L} \eta\left(\alpha+\sum_{m=1}^{k-1} \beta_{m}\right) T P\left|g_{k, l, i}\right|^{2}
$$

where $g_{k, l, i}$ is the channel coefficient of the link $\mathrm{PB}_{l} \rightarrow \mathrm{R}_{k, i}$, $\eta \in(0,1)$ presents the energy conversion efficiency, and $P$ denotes the transmit power of each power beacon. In the period of $\beta_{k}$, the transmit power of relay at hop $k$ is expressed as

$$
P_{k, i}=\frac{E_{k, i}}{\beta_{k} T}=\sum_{l=1}^{L} \chi_{k} P\left|g_{k, l, i}\right|^{2}
$$

where $\chi_{k} \triangleq \eta\left(\alpha+\sum_{m=1}^{k-1} \beta_{m}\right) / \beta_{k}$.

In the first-hop transmission, the maximal-ratio transmission beamforming [12] is employed at the source to transmit shortpackets to the relay $\mathrm{R}_{1, i}$ located in cluster 1 . The instantaneous signal-to-noise ratio (SNR) at $\mathrm{R}_{1, i}$ can be expressed as

$$
\gamma_{1, i}=\bar{\gamma}_{1}\left|\mathbf{h}_{1, i}^{H} \mathbf{w}\right|^{2} \text {, }
$$

where $\mathbf{w} \in \mathbb{C}^{N_{\mathrm{S}}}$ and $\mathbf{h}_{1, i} \in \mathbb{C}^{N_{\mathrm{S}}}$ represent the beamforming and channel coefficient vectors between $\mathrm{S}$ and $\mathrm{R}_{1, i}$, respectively, $\bar{\gamma}_{1} \triangleq P_{\mathrm{S}} / \sigma^{2}$, with $P_{\mathrm{S}}$ and $\sigma^{2}$ being transmit power at $S$ and the noise variance, respectively. Based on [6], the beamforming vector is designed based on the channel between $\mathrm{S}$ and $\mathrm{R}_{1, i}$, which is formulated as $\mathbf{w}=\mathbf{h}_{1, i} /\left\|\mathbf{h}_{1, i}\right\|$.

\section{B. Best Relay-Best User Scheme}

The bR-bU scheme selects the best relay as well as user providing the highest channel gain from the transmitter for packet transmission and reception, respectively. In the first hop, the instantaneous SNR at $\mathrm{R}_{1, i^{*}}$ can be presented as

$$
\gamma_{1}=\max _{i=1, \ldots, N_{1}} \bar{\gamma}_{1}\left|\mathbf{h}_{1, i}^{H} \mathbf{w}\right|^{2}=\max _{i=1, \ldots, N_{1}} \bar{\gamma}_{1}\left\|\mathbf{h}_{1, i}\right\|^{2} .
$$


For the remaining hops, we assume that $\mathrm{R}_{k, i^{*}}$ is the best relay at cluster $k$; thus, the relay at the cluster $k+1$ is selected by the following criterion:

$$
\mathrm{R}_{k+1, j^{*}}=\arg \max _{j=1, \ldots, N_{k+1}} \chi_{k} \bar{\gamma} \sum_{l=1}^{L} P\left|g_{k, l, i^{*}}\right|^{2}\left|h_{k, i^{*}, j}\right|^{2},
$$

where $\bar{\gamma} \triangleq P / \sigma^{2}$. The instantaneous SNR at hop $k+1$ can be expressed as

$$
\gamma_{k}=\max _{j=1, \ldots, N_{k+1}} \chi_{k} \bar{\gamma} \sum_{l=1}^{L} P\left|g_{l, k, i^{*}}\right|^{2}\left|h_{k, i^{*}, j}\right|^{2} .
$$

The outstanding performance of the proposed bR-bU for SPCs over benchmark schemes will be demonstrated in Section V.

\section{Performance AnAlysis}

Considering SPC at hop $k$, where a packet of $m$ information bits (message size) at $\mathrm{R}_{k-1, i}$ is transmitted to $\mathrm{R}_{k, j}$ over $\beta_{k}$ channel uses, with $\beta_{k}>100$ [4], received SNR equals to $\gamma_{k}$, and a transmission rate given by $r_{k}=m / \beta_{k}$. Thus, the average BLER at hop $k$ can be calculated as [3]

$$
\varepsilon_{k}\left(\gamma_{k}\right)=\mathbb{E}\left\{Q\left(\frac{C\left(\gamma_{k}\right)-r_{k}}{\sqrt{V\left(\gamma_{k}\right) / \beta_{k}}}\right)\right\}
$$

where $Q($.$) denotes the Gaussian Q-function,$ $C(x) \triangleq \log _{2}(1+x)$ is the Shannon capacity, and $V(x) \triangleq\left(1-\frac{1}{(1+x)^{2}}\right)\left(\log _{2} e\right)^{2}$ is the channel dispersion.

Directly evaluating an exact closed-form expression for $\varepsilon_{k}$ in (7) is very difficult since it involves a complicated Gaussian Q-function. To tackle this problem, we apply a tight approximation approach to derive the end-to-end (e2e) BLER, which will be presented in the next subsection.

\section{A. BLER Analysis}

From (4) and (6), the BLER of bR-bU scheme can be calculated by the following theorem.

Theorem 1. The closed-form expression for the BLER of $b R$ bU scheme can be derived as

$$
\varepsilon_{e 2 e} \approx 1-\left[1-\varepsilon_{1}\right] \prod_{k=2}^{K}\left[1-\varepsilon_{k}\right],
$$

where $\varepsilon_{1}$ and $\varepsilon_{k}$ are written, respectively, as

$$
\begin{aligned}
\varepsilon_{1} & =1+\delta_{1} \sqrt{\beta_{1}} \sum_{t=1}^{N_{1}} \sum_{q=0}^{t\left(N_{S}-1\right)}(-1)^{t}\left(\begin{array}{c}
N_{1} \\
t
\end{array}\right) \frac{b_{q}^{t} \bar{\gamma}_{1} \lambda_{D, 1}}{t^{q+1}} \\
\times & {\left[\gamma\left(1+q, t v_{1}\left(\bar{\gamma}_{1} \lambda_{D, 1}\right)^{-1}\right)-\gamma\left(1+q, t u_{1}\left(\bar{\gamma}_{1} \lambda_{D, 1}\right)^{-1}\right)\right], } \\
\varepsilon_{k} & =1-\delta_{k} \sqrt{\beta_{k}} \sum_{t=1}^{N_{k}}(-1)^{t-1}\left(\begin{array}{c}
N_{k} \\
t
\end{array}\right) \frac{\lambda_{D, k} \lambda_{E, k}}{t \Gamma(L)\left(\chi_{k} \bar{\gamma}\right)^{-1}} \\
& \times\left[G_{1,3}^{2,1}\left(\frac{t u_{k}\left(\chi_{k} \bar{\gamma}\right)^{-1}}{\lambda_{D, k} \lambda_{E, k}} \mid \begin{array}{c}
1 \\
1
\end{array}\right)\right. \\
& \left.-G_{1,3}^{2,1}\left(\frac{t v_{k}\left(\chi_{k} \bar{\gamma}\right)^{-1}}{\lambda_{D, k} \lambda_{E, k}} \mid L+1,1,0\right)\right],
\end{aligned}
$$

where $v_{k}, u_{k}, \delta_{k}$, and $\zeta_{k}$ in (10) can be presented, respectively, as $v_{k}=\zeta_{k}-1 /\left(2 \delta_{k} \sqrt{\beta_{k}}\right), u_{k}=\zeta_{k}+1 /\left(2 \delta_{k} \sqrt{\beta_{k}}\right)$, $\delta_{k}=\left[2 \pi\left(2^{2 r_{k}}-1\right)\right]^{-1 / 2}$, and $\zeta_{k}=2^{r_{k}}-1$, while these parameters in (9) can be obtained by giving the index $k=1$.
Proof: Please, see Appendix A.

As shown in (9) and (10), the BLER of bR-bU scheme is expressed under special Meijer's G-functions, which may be difficult to draw some insights from a non-expert in the field. This motivates us to perform an asymptotic analysis, which results in simpler expressions.

\section{B. Asymptotic BLER at High SNR}

Theorem 2. The asymptotic expression for the BLER of $b R$ bU scheme when $\bar{\gamma} \rightarrow \infty$ can be derived as

$$
\varepsilon_{e 2 e}^{\infty} \approx F_{\gamma_{1}}\left(\zeta_{k}\right)+\sum_{k=2}^{K} F_{\gamma_{k}}\left(\zeta_{k}\right)
$$

where $F_{\gamma_{1}}($.$) and F_{\gamma_{k}}($.$) are given in (19) and (21), respec-$ tively.

Proof: Please, see Appendix B.

\section{Throughput Analysis}

We consider the delay-limited transmission mode, where the throughput is calculated by using the e2e BLER. For a fixed data rate $R_{t h}$ bits per channel use (BPCU), and the effective communication time $\frac{(\omega-\alpha) T}{K}$ over total transmission time $\omega T$, the effective e2e throughput can be expressed as

$$
\tau=\frac{R_{t h}(\omega-\alpha)\left(1-\varepsilon_{e 2 e}\right)}{\omega K} .
$$

To support real-time settings, we design a DNN model for throughput prediction in the next section.

\section{Deep Learning Design for The Throughput PREDICTION}

\section{A. Description of DNN}

Fig. 3 illustrates the architecture of the designed DNN model to predict the throughput of bR-bU scheme, which consists of multiple hidden layers between an input and an output layers. The related system variables with their ranges, which comprise of the number of hops with $K \in[1,6]$, the number of antennas at $\mathrm{S}$ with $N_{\mathrm{S}} \in[1,5]$, the number of PBs with $L \in[1,4]$, the number of relays in each cluster with $N_{k} \in[1,5]$, the positions of PBs with $x_{\mathrm{PB}} \in[6,9]$ and $y_{\mathrm{PB}} \in[6,9]$, the average SNR with $\bar{\gamma} \in[1,30]$, the channel uses for WET with $\alpha \in[100,200]$, and the target data rate with $R_{t h} \in[1,3]$, are used as the input variables of DNN. The throughput is generated based on (12) which is the output of DNN. Based on these setups, the whole dataset having 120000 samples is divided randomly into the training and test sets with the ratio of $80-20$. We have numerically observed that this number of samples is sufficient to obtain high accurate predictions in most cases.

\section{B. The Learning Model}

In each hidden layer, the rectified linear unit (ReLU) activation function is used to perform a threshold operation to every input element [10]. Compared with the Sigmoid or Tanh activations, the ReLU activation provides significant benefits such as computational simplicity, easier to optimize 


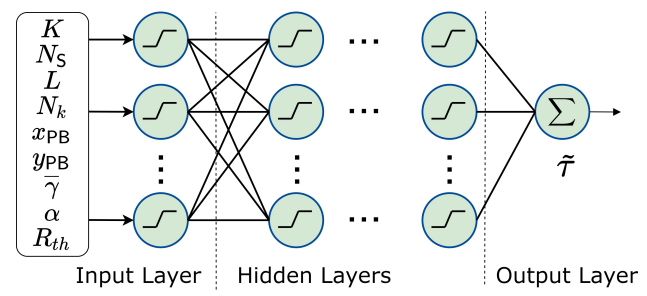

Fig. 3. The proposed DNN model for throughput prediction.

due to its near-linear behavior, and avoiding the problem of vanishing gradients [13]. In this DNN model, the output layer has no activation function because the regression model can directly output a value without using any transformations. We present the activation process at the $r$-th neuron of $p$-th layer connecting to that of $(p-1)$-th layer as follows:

$$
a_{r}^{p}=\operatorname{ReLU}\left(\sum_{q=1}^{Q_{p-1}} w_{r q}^{p} a_{q}^{p-1}+b_{r}^{p}\right),
$$

where $w_{r q}^{p}$ is the weight connected to the $q$-th neuron in the $(p-1)$-th layer, $b_{r}^{p}$ is the scalar bias at $p$-th layer, and $Q_{p-1}$ is the number of neurons in the $(p-1)$-th layer. For the regression problem, the loss function indicating the error between predicted and expected values, which can be expressed as

$$
\mathcal{L}(\Delta)=\frac{1}{\Delta} \sum_{m=1}^{\Delta}\left(\tau_{(m)}-\tilde{\tau}_{(m)}\right)^{2},
$$

where $\Delta$ is the number of training samples, $\tau_{(m)}$ and $\tilde{\tau}_{(m)}$ are the expected and predicted values, respectively. We apply the adaptive moment estimation optimization algorithm for the backpropagation procedure to update the weights and biases, which aims at minimizing the loss function of the entire training set.

\section{Real-Time Prediction}

After completing the offline training, the resulting DNN model can be presented under a low-latency inference mapping function as $\mathcal{F}($.$) . In general, when the DNN is well trained,$ it can provide real-time and highly accurate predictions. We use the obtained DNN model to predict the throughput value whenever new information is available at the input. In particular, we arrange each input sample into a vector $\mathbf{x} \triangleq\left[K, N_{\mathrm{S}}, L, N_{k}, x_{\mathrm{PB}}, y_{\mathrm{PB}}, \bar{\gamma}, \alpha, R_{t h}\right]$ and it is placed at the input of the DNN, a predicted throughput will appear at the output, which is presented as

$$
\tilde{\tau}=\mathcal{F}(\mathbf{x}) \text {. }
$$

Through a low-latency inference process in (15), the system throughput can be predicted by the DNN model with a short time. By adaptively adding more hidden layers or more neurons into the designed DNN, the training error can be reduced without occurring overfitting. If the predicted throughput is not close to the actual one, the DNN will need to be retrained with new appropriate settings (new learning rate, the number of hidden layers, and hidden neurons) until achieving the smallest error in (14).

\section{NumERICAL RESUlTS AND DiscusSiONS}

In this section, numerical examples are presented to evaluate the achievable performance of the proposed relay selection schemes. Monte-Carlo simulations are used to verify our analysis results. A bi-dimensional plane is considered, where the simulation variables are set as follows: $d_{\mathrm{SD}}=30 \mathrm{~m}$, $d_{0}=1 \mathrm{~m}, \mathcal{L}=-30 \mathrm{~dB}, \sigma_{\mathrm{PL}}=3, \eta=0.8, \omega=1000$, $m=1024$ bits, $\alpha=100, N_{\mathrm{S}}=3, L=2, P=P_{\mathrm{S}}$, $K=5, N_{k}=3, R_{t h}=1$, and $\sigma^{2}=1$. The position of $\mathrm{S}$, $\mathrm{R}_{k, i}, \mathrm{D}$, and $\mathrm{PB}$ are $(0,0),(k / K, 0),(30,0)$, and $(7.0,7.0)$, respectively. We implement the DNN with 4 hidden layers and 170 hidden neurons in Python 3.7.4 with Keras 2.3.1 using TensorFlow 2.0.0, where is end-to-end trained in 70 epochs with the initial learning rate as $10^{-3}$ (dropped $90 \%$ after 10 epochs). The RMSE is calculated over the whole test set and it can be presented as RMSE $=\sqrt{\mathcal{L}(\widetilde{\Delta})}$, where $\widetilde{\Delta}$ denotes the number of samples in the test set. To demonstrate the excellent performance of the bR-bU scheme, we consider three benchmark schemes as follows:

- "Random Relay-Random User (rR-rU)": The rR-rU scheme randomly selects the relay as well as user for packet transmission and reception, respectively.

- "Best Relay-Random User (bR-rU)": The bR-rU scheme selects the best relay providing the highest SNR from the transmitter for packet transmission while randomly choosing a user for packet reception.

- "Random Relay-Best User (rR-bU)": This scheme randomly selects a relay for packet transmission while picking the best user having the highest SNR from the transmitter for packet reception.

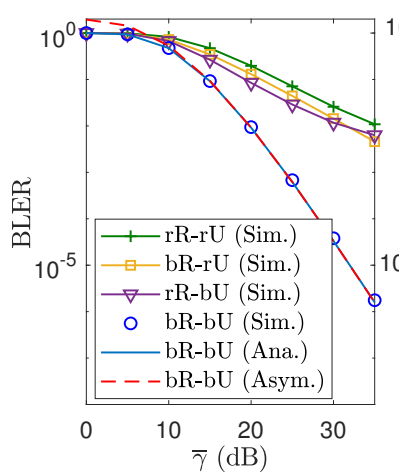

(a)

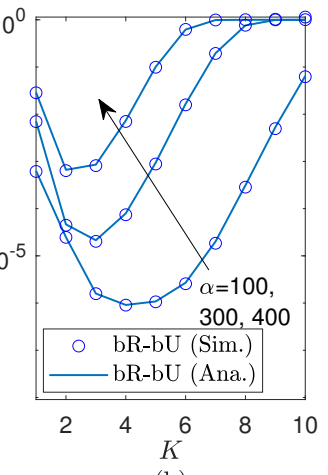

(b)
Fig. 4. Average BLER versus $\bar{\gamma}$ and the number of hops with different $\alpha$.

As shown in Fig. 4(a), the bR-bU scheme provides better BLER than baseline schemes such as rR-rU, bR-rU, and rRbU. Multiple relays and users jointly involving in the relay selection process enable the bR-bU scheme to achieve high diversity and reliable transmissions while deploying singlerelay or single-user selection in baseline schemes results in poor BLER performance. The effect of the number of hops on the BLER is shown in Fig. 4(b), where the difference of $\alpha$ values gives different number of optimal hops. However, when 
$K$ is large, the BLER gets worse, showing that the effect of $K$ is more important than that of $\alpha$. The analytical results match perfectly with the simulation ones while the asymptotic results are tight upper bounds, confirming results in Theorems 1 and 2 , respectively.

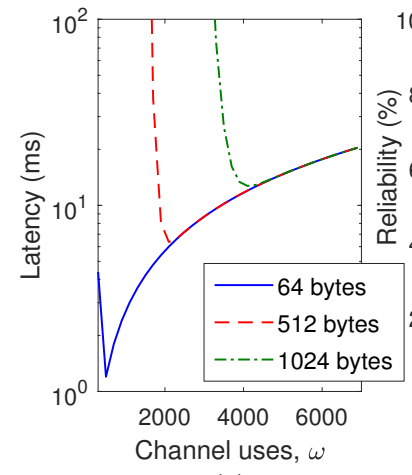

(a)

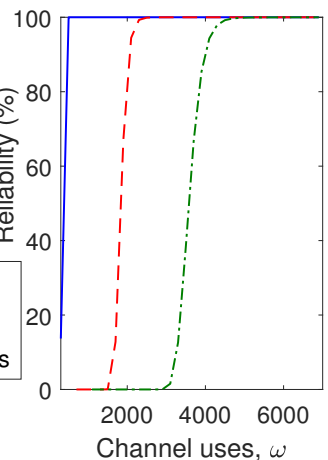

(b)
Fig. 5. The latency and reliability of short and long messages.

In Fig. 5, the short-messages for SPCs provide lower latency and higher reliability than the long-message ones. The latency and reliability can be defined, respectively, as $\frac{(\omega-\alpha) T}{1-\varepsilon_{e 2 e}}$ and $\left(1-\varepsilon_{e 2 e}\right) \times 100 \%$, with $T=3 \mu s$ [3], [14]. The 64-byte messages have lower latency and higher reliability than 512 and 1024-byte ones. To guarantee the reliability, these long messages can be framed into packets with more than 4000 channel uses by using channel coding schemes [1], their latencies are still high and can not meet the requirement of URLLC applications, e.g., in excess of $10 \mathrm{~ms}$ to deliver a 1024-byte packet, as shown in Fig. 5(b). Therefore, longmessages can not meet the constraint of latency, whereas the SPCs with short-messages can attain either latency or reliability requirement.

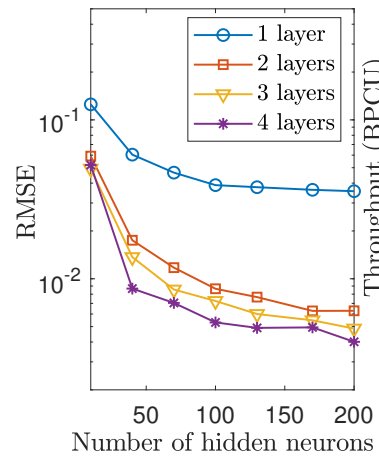

(a)

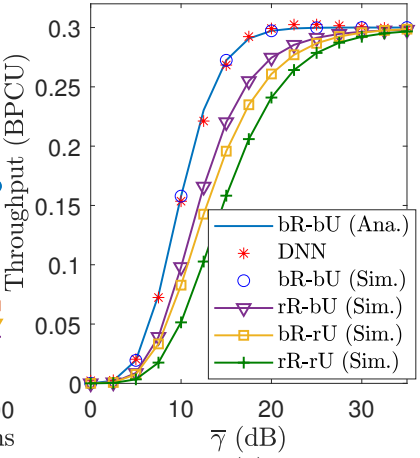

(b)
Fig. 6. RMSE of the DNN model and throughput versus $\bar{\gamma}$.

In Fig. 6(a), we plot the RMSE with different hidden layers to show some insights into the DNN design. As can be observed, the more hidden layers embedded in the DNN, the lower the RMSE, and the higher accuracy the predicted

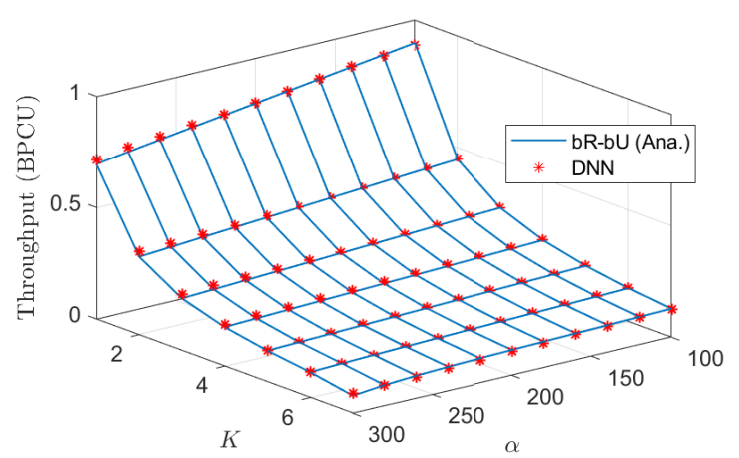

Fig. 7. Throughput versus $\alpha$ and $K$ with $\bar{\gamma}=30$ (dB).

throughput is. It is clear that multiple hidden layers employed for the DNN can generalize dataset, leading to high neural network capacity, whilst the single-layer is incapable of learning the complicated patterns of dataset, resulting in the high RMSE. Fig. 6(b) shows that the throughput of all schemes is improved as $\bar{\gamma}$ increases since the relays have more opportunities to harvest a sufficient amount of energy from PBs, resulting in the throughput enhancement. Moreover, the bR-bU scheme provides the best performance while the rR-rU scheme is the lowest performer. There is an excellent agreement among Monte-Carlo simulations, theoretical analyses, and DNN prediction results, verifying our designed approaches.

Fig. 7 illustrates the effect of the number of hops, $K$, and channel uses for WET, $\alpha$, on the throughput of bR-bU scheme. When $K$ increases, the BLER for packet transmission is large due to the pathloss effect; thus, the throughout is reduced as in (12). When $\alpha$ increases, more channel uses for data transmission are required by the network. Moreover, DNN prediction results coincide with the throughput of bR-bU ones in most cases, verifying our effective design of the DNN model.

TABLE I

THE COMPARISON OF EXECUTION TIME AMONG SIMULATION (Sim.), THEORETICAL ANALYSES BASED ON THEOREM 1 (Theo.), AND DNN PREDICTION (DNN), WITH $\bar{\gamma}=20 \mathrm{~dB}$.

\begin{tabular}{|c|c|c|c|c|}
\hline Scenarios $\left\{L, N_{k}, K\right\}$ & Sim. & Theo. & DNN & RMSE \\
\hline$\{2,3,4\}$ & $10.872 \mathrm{~s}$ & $01.800 \mathrm{~s}$ & $0.0279 \mathrm{~s}$ & $1.5751 \times 10^{-4}$ \\
\hline$\{3,4,5\}$ & $17.276 \mathrm{~s}$ & $02.377 \mathrm{~s}$ & $0.0299 \mathrm{~s}$ & $2.4 \times 10^{-3}$ \\
\hline$\{4,5,6\}$ & $24.124 \mathrm{~s}$ & $03.014 \mathrm{~s}$ & $0.0319 \mathrm{~s}$ & $4.3168 \times 10^{-4}$ \\
\hline
\end{tabular}

Finally, the execution time of the throughput evaluation of bR-bU scheme is shown in Table I, in which each sample in the first column (Sim.) is averaged with $5 \times 10^{5}$ trials using of Monte-Carlo simulations. These results show that the simulation requires the longest time while the DNN model is found to have the fastest execution time, which enables a novel DNN-based real-time setting in practical WPINs. 


\section{CONCLUSIONS}

In this paper, we proposed a bR-bU scheme to improve the BLER and throughput performance of WPINs with SPCs. The asymptotic BLER was also evaluated in a tractable form which was shown to be a tight upper bound for the BLER at high SNR regime. The DNN model was designed for the bR-bU scheme to predict its throughput. Numerical results demonstrated the BLER and throughput improvements of bRbU scheme over several baseline ones. Moreover, the DNN model accurately predicted the throughput with low execution time. These results advocated the proposed DNN method for real-time solution in future WPINs with SPCs.

\section{APPENDIX A}

PROOF OF THEOREM 1

First, the BLER at hop $k$ can be rewritten from (7) as

$$
\varepsilon_{k}=\int_{0}^{\infty}\left[Q\left(\frac{C\left(\gamma_{k}\right)-r_{k}}{\sqrt{V\left(\gamma_{k}\right) / \beta_{k}}}\right)\right] f_{\gamma_{k}}(x) d x
$$

In order to calculate the integral (16), we apply a linear approximation for Q-function as $Q\left(\frac{C\left(\gamma_{k}\right)-r_{k}}{\sqrt{V\left(\gamma_{k}\right) / \beta_{k}}}\right) \approx \Xi\left(\gamma_{k}\right)$, where $\Xi\left(\gamma_{k}\right)$ can be expressed as [3], [4]

$$
\Xi\left(\gamma_{k}\right)= \begin{cases}1, & \gamma_{k} \leq v_{k}, \\ 0.5-\delta_{k} \sqrt{\beta_{k}}\left(\gamma_{k}-\zeta_{k}\right), & v_{k} \leq \gamma_{k} \leq u_{k}, \\ 0, & \gamma_{k} \geq u_{k} .\end{cases}
$$

With the above approximation, we continue to apply the partial integration method, which yields

$$
\varepsilon_{k}=\delta_{k} \sqrt{\beta_{k}} \int_{v_{k}}^{u_{k}} F_{\gamma_{k}}(x) d x .
$$

We can now proceed with the derivation for the CDFs of $\gamma_{1}$ and $\gamma_{k}$. Considering (4) with $\left\|\mathbf{h}_{1, i}\right\|$ being a Gamma random variable (RV), and consequently $\gamma_{1}$ is the maximum of $N_{k}$ Gamma RVs. Thus, the CDF of $\gamma_{1}$ is given by [6, Eq. (25)]

$$
F_{\gamma_{1}}(x)=1+\sum_{t=1}^{N_{1}} \sum_{q=0}^{t\left(N_{S}-1\right)}(-1)^{t}\left(\begin{array}{c}
N_{1} \\
t
\end{array}\right) b_{q}^{t}\left(\frac{\bar{\gamma}_{1}^{-1} x}{\lambda_{D, 1}}\right)^{q} \exp \left(-\frac{\bar{\gamma}_{1}^{-1} t x}{\lambda_{D, 1}}\right) \text {. }
$$

where coefficient $b_{q}^{t}$ is given in [6, Eq. (17)]. Plugging (19) into (18) with the index $k=1$, it follows that

$$
\varepsilon_{1}=1+\sum_{t=1}^{N_{1}} \sum_{q=0}^{t\left(N_{S}-1\right)}(-1)^{t}\left(\begin{array}{c}
N_{1} \\
t
\end{array}\right) b_{q}^{t} \int_{v_{k}}^{u_{k}}\left(\frac{x \bar{\gamma}^{-1}}{\lambda_{D, 1}}\right) \exp \left(-\frac{x \bar{\gamma}^{-1}}{\lambda_{D, 1}}\right) d x \text {. }
$$

After solving the integral in (20), $\varepsilon_{1}$ can be obtained as shown in (9). Next, the CDF of $\gamma_{k}$ can be given as [7, Eq. (13)]

$$
\begin{aligned}
F_{\gamma_{k}}(x)= & -\sum_{t=1}^{N_{k}} \frac{2(-1)^{t-1}}{\Gamma(L)}\left(\begin{array}{c}
N_{k} \\
t
\end{array}\right)\left(\frac{t x}{\chi_{k} \lambda_{D, k} \lambda_{E, k} \bar{\gamma}}\right)^{\frac{L}{2}} \\
& \times K_{L}\left(2 \sqrt{\frac{t x}{\chi_{k} \lambda_{D, k} \lambda_{E, k} \bar{\gamma}}}\right) .
\end{aligned}
$$

Then, substituting (21) into (18), then applying [11, Eq. (9.34.3)] to represent $K_{v}($.$) function in terms of Meijer's G-$ function, thus yielding

$\varepsilon_{k}=1-\sum_{t=1}^{N_{k}} \frac{2^{1-L} \delta_{k} \sqrt{\beta_{k}}}{(-1)^{1-t} \Gamma(L)}\left(\begin{array}{c}N_{k} \\ t\end{array}\right) \int_{v_{k}}^{u_{k}} G_{0,2}^{2,0}\left(\frac{t x\left(\chi_{k} \bar{\gamma}\right)^{-1}}{\lambda_{D, k} \lambda_{E, k}} \mid L, 0\right) d x$.
Finally, the integral (22) can be solved by using the results in [15], where $\varepsilon_{k}$ can be obtained as shown in (10).

\section{APPENDIX B}

\section{PRoOF of THEOREM 2}

By utilizing the first order Riemann integral approximation, i.e., $\int_{x}^{y} f(z) d z=(y-x) f\left(\frac{x+y}{2}\right)$ for (18), we obtain the average BLER at hop $k$ as

$$
\varepsilon_{k}=F_{\gamma_{k}}\left(\zeta_{k}\right) .
$$

Furthermore, the asymptotic expression for the BLER of bRbU scheme can be attained by invoking the following expression: $\prod_{k=1}^{K}\left(1-x_{k}\right) \approx 1-\sum_{k=1}^{K} x_{k}$, which is valid for small $x_{k}$. Finally, from (8) and (23), the asymptotic expression for the BLER of bR-bU scheme can be asymptotically expressed as (11), which concludes the proof of Theorem 2.

\section{ACKNOWLEDGMENT}

This work was supported by National Research Foundation of Korea (NRF) grant funded by the Korea government (MSIT) (NRF-2019R1A2C1083996).

Prof. Beongku An is the corresponding author.

\section{REFERENCES}

[1] I. Parvez, A. Rahmati, I. Guvenc, A. I. Sarwat, and H. Dai, "A survey on low latency towards 5G: RAN, core network and caching solutions,' IEEE Commun. Surv. Tutor, vol. 20, no. 4, pp. 3098-3130, 4th Quart. 2018.

[2] T. Hößler, P. Schulz, E. A. Jorswieck, M. Simsek, and G. P. Fettweis, "Stable matching for wireless URLLC in multi-cellular, multi-user systems," IEEE Trans. Commun., vol. 68, no. 8, pp. 5228-5241, May 2020.

[3] O. L. A. López, H. Alves, R. D. Souza, and E. M. G. Fernández, "U1trareliable short-packet communications with wireless energy transfer," IEEE Signal Process. Lett., vol. 24, no. 4, pp. 387-391, Apr. 2017.

[4] B. Makki, T. Svensson, and M. Zorzi, "Wireless energy and information transmission using feedback: Infinite and finite block-length analysis," IEEE Trans. Commun., vol. 64, no. 12, pp. 5304-5318, Dec. 2016.

[5] J. Chen, L. Zhang, Y.-C. Liang, X. Kang, and R. Zhang, "Resource allocation for wireless-powered IoT networks with short packet communication," IEEE Trans. Wireless Commun., vol. 18, no. 2, pp. 1447-1461, Feb. 2019.

[6] T.-V. Nguyen and B. An, "Cognitive multihop wireless powered relaying networks over Nakagami- $m$ fading channels," IEEE Access, vol. 7, pp. $154600-154616$, Oct. 2019.

[7] T.-V. Nguyen, T.-N. Do, V. N. Q. Bao, D. B. da Costa, and B. An, "Performance analysis of multihop cognitive WPCNs with imperfect CSI," in 2019 IEEE Global Commun. Conf. (GLOBECOM), Waikoloa, HI, USA, Dec. 2019, pp. 1-6.

[8] Y. Gu, H. Chen, Y. Li, and B. Vucetic, "Ultra-reliable short-packet communications: Half-duplex or full-duplex relaying?" IEEE Wireless Commun. Lett., vol. 7, no. 3, pp. 348-351, Jun. 2018.

[9] Q. Mao, F. Hu, and Q. Hao, "Deep learning for intelligent wireless networks: A comprehensive survey," IEEE Commun. Surv. Tutor., vol. 20, no. 4, pp. 2595-2621, 4th Quart. 2018.

[10] T.-V. Nguyen, T.-N. Tran, K. Shim, T. Huynh-The, and B. An, "A deep neural network-based relay selection scheme in wireless-powered cognitive IoT networks," IEEE Internet Things J., Nov. 2020.

[11] I. S. Gradshteyn and I. M. Ryzhik, Table of integrals, series, and products. Academic Press, 2007.

[12] T. K. Lo, "Maximum ratio transmission," in Proc. IEEE Int. Conf. Commun., vol. 2. IEEE, Jun. 1999, pp. 1310-1314.

[13] X. Glorot, A. Bordes, and Y. Bengio, "Deep sparse rectifier neural networks," in Proc. 14th Int. Conf. Artificial Intelligence and Statistics (AISTATS), vol. 15, Fort Lauderdale, FL, USA, Jun. 2011, pp. 315-323.

[14] R. Devassy, G. Durisi, P. Popovski, and E. G. Ström, "Finite-blocklength analysis of the ARQ-protocol throughput over the gaussian collision channel," in 2014 6th Int. Symposium Commun., Control and Signal Processing (ISCCSP), Athens, Greece. IEEE, Aug. 2014, pp. 173-177.

[15] W. F. Site, MeijerG. [Online]. Available: http://functions. wolfram.com/07.34.21.0002.01, 2001. 